\title{
Die Rechtsfrage:
}

\section{Wann handelt es sich um freie Mitarbeit und wann um Schein- selbstständigkeit?}

D. Benjamin Alt ist niedergelassener Rechtsanwalt und mit seinem Team bundesweit maßgeblich im Bereich Medizinrecht, Wettbewerbsrecht und Gesellschaftsrecht tätig. Er vertritt vor allem Heilmittelerbringer sowie Ärzte und ist Justiziar des Verbands Physikalische Therapie (VPT). Alt veröffentlichte mehrere Fachbücher und ist bekannt für seine umfassenden Dozententätigkeiten.

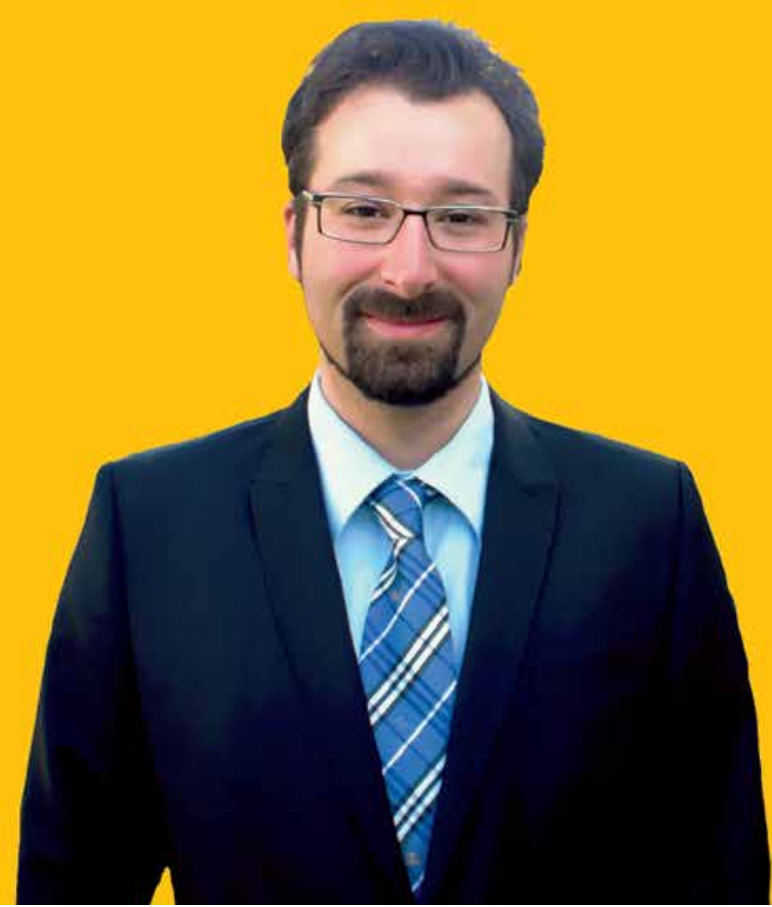

2014 gab es mehrere Urteile von Landessozialgerichten, die Therapeuten und Praxisinhaber verunsicherten: Ist freie Mitarbeit in einer Praxis erlaubt? Kürzlich hat das Bundessozialgericht sich klar dazu geäußert, dass dies rechtlich zulässig ist. Wichtig ist, dass der freie Mitarbeiter die Kriterien der selbstständigen Tätigkeit erfüllt. physiopraxis sprach darüber mit Rechtsanwalt D. Benjamin Alt.

Herr Alt, viele Therapeuten sind verunsichert. Sie befinden sich auf einer Gratwanderung zwischen Scheinselbstständigkeit und freier Mitarbeit. Wo genau sind dabei die Grenzen? Wesentliches Kriterium ist die Eingliederung in den Betrieb. Sofern sich

\section{7} ein Mitarbeiter in den Betrieb eingliedern lässt und er von den Arbeitnehmern nicht zu unterscheiden ist, kann man üblicherweise davon ausgehen, dass er scheinselbstständig ist. Für eine Scheinselbstständigkeit spricht, dass der Therapeut nicht selbst mit den Patienten abrechnet, er keinen festen
Behandlungsraum hat und die Terminvergabe über das Praxispersonal stattfindet. Diese Punkte sollten möglichst vermieden werden. Im Rahmen der Abrechnung mit den gesetzlichen Krankenkassen stellt dies jedoch dann schon eine unüberwindbare Hürde dar, weil der freie Mitarbeiter selber nicht mit den gesetzlichen Krankenkassen abrechnen kann. Er muss seine Verordnungen über den Praxisinhaber abrechnen lassen.

Welche konkreten Kriterien müssen für die freie Mitarbeit in einer Praxis erfüllt sein?

Einen festen Kriterienkatalog gibt es nicht. Es hängt von der Gesamtsituation ab. Zeichnet sie eher das Bild einer freien Mitarbeiterschaft oder eher ein abhängiges Beschäftigungsverhältnis? Damit eine freie Mitarbeiterschaft anerkannt wird, ist es ratsam, dass der Therapeut nicht in den Praxisräumlichkeiten tätig ist, sondern nur auf Hausbesuchen. Dadurch wird er weniger als Angestellter angesehen werden können.
Des Weiteren ist es sinnvoll, dass er eigene Therapiegegenstände benutzt, eine eigene Berufshaftpflichtversicherung hat, selber Rentenversicherungsbeiträge zahlt und mit Privatpatienten selbst abrechnet. Ein deutliches Indiz für eine freie Mitarbeiterschaft wäre auch die Beschäftigung von eigenen Angestellten oder der Betrieb einer eigenen Privatpraxis. All dies sind jedoch nur Indizien, die eine vollständige Sicherheit nicht gewährleisten.

Hausbesuche müssen auch über den Praxisinhaber abgerechnet werden. Worin liegt der Unterschied zur Tätigkeit in der Praxis?

Der Unterschied liegt darin, dass der Therapeut, der nur Hausbesuche durchführt, weniger als Teil des Praxisteams angesehen werden kann. Er tritt dann nicht zusammen mit der Praxis auf, und es besteht bereits eine räumliche Trennung zwischen der Praxis und seiner therapeutischen Tätigkeit.

Zudem werden nicht wenige Patienten nur im Rahmen von Hausbesuchen behandelt. Diese haben dann nur eine Bindung zum konkreten Therapeuten. Die Praxis, für welche der Therapeut arbeitet, spielt in diesen Fällen dann gar keine Rolle. 
Wenn der freie Mitarbeiter an den Praxisinhaber beispielsweise für die Nutzung der Räume, der Rezeptionskraft und des Telefons bezahlt und selbst festlegt, wann seine Arbeitszeiten in der Praxis sind, hebt er sich doch eindeutig von der Situation eines Angestellten ab.

Das ist vollkommen richtig. In der Realität wird dies jedoch selten derart gehandhabt. Oft zeigt sich dies schon daran, dass die aufgezählten Leistungen üblicherweise umsatzsteuerpflichtig wären und der Praxisinhaber keine Rechnung an den freien Mitarbeiter stellt, auf welcher die genannten Positionen aufgelistet sind. Die Unterscheidung bei der tatsächlichen Arbeit im Vergleich zu Angestellten ist für die Rentenversicherung eben nicht das maßgebliche Kriterium. Viel wichtiger ist, ob der freie Mitarbeiter als Teil der Praxis angesehen wird und dort eingegliedert ist.

Wie kann ich als Praxisinhaber und als Therapeut die Kriterien für eine freie Mitarbeit prüfen? Gibt es eine sichere, objektive Instanz, an die ich mich wenden kann?

Das Wichtigste ist, dass man sich vor Aufnahme der Beschäftigung mit der sogenannten Clearingstelle der Deutschen Rentenversicherung in Verbindung setzt. Sie kann eine Statusfeststellung durchführen. Es handelt sich dabei um einen Antrag bei der Rentenversicherung, die im Einzelfall das Verhältnis zwischen der auftraggebenden Praxis sowie dem Mitarbeiter prüft. Diese Statusfeststellung gilt immer nur zwischen einer Praxis und einem freien Mitarbeiter und ist auf andere Praxen nicht übertragbar.

Stellt der Praxisinhaber einen solchen Antrag

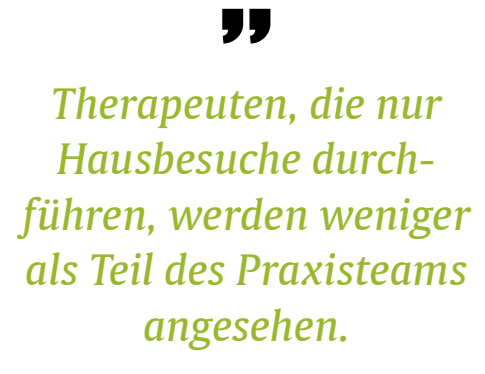

bei der Rentenversicherung, übersendet diese an die Praxis umfangreiche Formulare und Fragebögen. Hier lohnt es sich durchaus, eine anwaltliche Beratung in Anspruch zu nehmen, weil bei unbedarfter Beantwortung der Fragen eine negative Entscheidung der Deutschen Rentenversicherung zu erwarten ist. Diese kann hohe Sozialversicherungsbeiträge als Folge nach sich ziehen.

Würde die Rentenversicherung jedoch in dem Verfahren feststellen, dass eine freie Mitarbeiterschaft vorliegt, können sich alle Betei- ligten darauf verlassen. Nur für die Zukunft kann eine solche Feststellung zurückgenommen werden, aus der Vergangenheit können sich jedoch dann keine Ansprüche ergeben. Vorausgesetzt die Beteiligten halten sich auch an das, was sie der Rentenversicherung mitgeteilt haben. Dies gilt vor allem für die Regelungen, welche im Vertrag für den freien Mitarbeiter festgehalten wurden. Der Vertrag ist schließlich das zentrale Kriterium der Beurteilung und sollte professionell für den Einzelfall ausgearbeitet worden sein.

\section{Was kann ich tun, wenn ich merke, dass die Kriterien nicht erfüllt sind?}

Sofern bereits eine vermeintliche freie Mitarbeiterschaft eingegangen wurde und bereits über längere Zeit praktiziert wird, kann ich nur dazu raten, das Arbeitsverhältnis umgehend umzustellen. Ist dies nicht der Fall, muss der Praxisinhaber mit hohen Nachforderungen rechnen. Findet die Rentenversicherung bei einer Betriebsprüfung heraus, dass ein Mitarbeiter eigentlich scheinselbstständig ist, muss in etwa mit 25 Prozent Nachforderung der Summe gerechnet werden, die der Praxisinhaber an den freien Mitarbeiter als Honorar bezahlt hat. Es spielt dabei nicht unbedingt eine Rolle, ob dieser selbst schon Beiträge an die Rentenversicherung abgeführt hat. Die Rückforderungsansprüche der Rentenversicherung verjähren übrigens üblicherweise nach fünf Jahren.

Die einfachste Lösungsmöglichkeit ist hier selbstverständlich, dass der Praxisinhaber den Mitarbeiter anstellt. Dann besteht keine Gefahr mehr. Eine wohl im Einzelfall sinnvollere Lösung ist die Gründung einer sogenannten SeniorJunior-Partnerschaft, in der sich die bisherige Praxis und der freie Mitarbeiter in Form einer Gesellschaft zusammenschließen. Dies ist wesentlich unkomplizierter, als es sich zunächst anhört. Hier muss für den Einzelfall ein Gesellschaftsvertrag erstellt werden, welcher dazu führen soll, dass im Wesentlichen die Aufgaben und Risikoverteilungen so sind wie in der freien Mitarbeiterschaft angedacht. In weiten Teilen kann dies auch vertraglich geregelt werden. Ich habe schon sehr viele Praxen bei der Gründung einer solchen Gesellschaft unterstützt. Dabei kann eine Regelung geschaffen werden, die der freien Mitarbeiterschaft sehr nahe kommt und weder große Risiken für den Praxis-

inhaber noch den ehemals freien Mitarbeiter darstellt.

In anderen Branchen ist die freie Mitarbeit üblich - können wir diesbezüglich auf eine positive Entwicklung auch im Bereich der Heilmittelerbringer hoffen?

Es ist damit zu rechnen, dass auch in anderen Branchen viele Prüfungen in den kommenden Jahren stattfinden werden. Die Rentenversicherung hat sich teils in den letzten Jahren auf Physio- und +therapeuten eingeschossen, weil dort viele freie Mitarbeiter tätig sind und seitens der Rentenversicherung eine hohe Trefferquote besteht, dass man Nachzahlungen geltend machen kann. In diesen Fällen ist die Trefferquote so hoch, weil eben durch den Therapeuten ein direkter Kontakt zum Patienten besteht. In Branchen, in denen kein Kontakt zum Endkunden besteht, sind die Chancen wesentlich größer, dass eine freie Mitarbeiterschaft von der Rentenversicherung akzeptiert wird. Die Fragen stellte Nicole Kaldewei.

\section{Ihre Erfahrung}

\section{Freie Mitarbeit in der Praxis}

Austausch erwünscht. Arbeiten Sie als freier Mitarbeiter in einer Praxis und haben Ihre Statusprüfung in trockenen Tüchern? Dann berichten Sie uns über Ihre Erfahrungen. An konkreten Beispielen aus der Praxis lässt sich am besten lernen. Schreiben Sie einfach an physiopraxis@thieme.de. 